\title{
Stepped care for depression in primary care: what should be offered and how?
}

\author{
Annemieke van Straten, Wike Seekles, Nelleke J van 't Veer-Tazelaar, Aartjan T F Beekman and Pim Cuijpers
}

$\longrightarrow$ urrently, major depression is one of the most debilitating disorders in Western countries due to its high prevalence and burden of disease. ${ }^{1,2}$ Although effective treatments and clinical guidelines are available in most Western countries, a number of obstacles exist with respect to accessibility, effectiveness and efficiency in the management of depression. First, a large number of patients are not treated at all. It is estimated that about a third to half of all cases of depression, both mild and severe, are not recognised as such. ${ }^{3-5}$ Second, even when depression is recognised, treatment is not always based on evidence. Although precise estimates are lacking, it has been suggested that this is true for as many as $50 \%$ of patients. ${ }^{6}$

The recognition of these and other obstacles in depression management has led to alternative methods of treatment delivery. Most well known service models are based on the constructs of collaborative care, disease management and stepped care. ${ }^{7,8}$ These models usually include a number of different evidence-based interventions, close involvement of non-medical specialists, monitoring of outcomes, and the use of care managers. In many collaborative care and disease management programs, assignment of the available treatment options is based on interdisciplinary discussions of the patient's needs. As a result, different patients start with different treatments.

In stepped-care models, all patients start with the same lowintensity intervention. Progress is monitored, and only those patients who do not respond adequately step up to a subsequent intervention of higher intensity. The assumption of stepped care is that many patients benefit from low-intensity treatments and do not need further treatment. In theory, this means that stepped care is the most cost-efficient model of care delivery. It might be a way to meet increasing care demands and to improve allocation of scarce resources. Stepped-care models have been described for disorders such as weight control, eating disorders, generalised anxiety disorder, panic disorder, and alcohol problems. ${ }^{9-14}$ Although the first results seem promising, large-scale, good-quality randomised trials into the effectiveness and cost-effectiveness of full stepped-care models are still lacking. ${ }^{15}$

Being a low-intensity treatment, guided self-help fits very well within stepped care. In recent years, self-help has been increasingly offered through the internet, and it has been convincingly demonstrated to be effective in reducing mental health problems in the general population. ${ }^{16-19}$ Evidence about effectiveness in primary care patients is also starting to emerge (see Hickie et al, page S31). ${ }^{20,21}$ However, not all patients want to start with selfhelp, whether web-based or not, nor will every patient benefit from it. Therefore, it is not recommended to offer self-help as a stand-alone therapy but to include it within a more comprehensive care model, such as the stepped-care model.

Here, we describe the development and evaluation of a steppedcare model implemented in the Netherlands. We will illustrate this using knowledge gained from two randomised trials: the first was aimed at older patients (75 years or older) with subthreshold depression and anxiety disorders; and the second targeted younger
ABSTRACT

- Stepped-care approaches may offer a solution to delivering accessible, effective and efficient services for individuals with depression.

- In stepped care, all patients commence with a low-intensity, low-cost treatment. Treatment results are monitored systematically, and patients move to a higher-intensity treatment only if necessary.

- We deliver a stepped-care model targeting patients with depression.

> The first step consists of "watchful waiting", as half of all patients with a depressive episode recover spontaneously within 3 months.

$>$ The second step, guided self-help, is the key element of the stepped-care model. Guided self-help, especially when offered through the internet, is effective and cost-efficient.

$>$ The third step consists of brief face-to-face psychotherapy.

$>$ Finally, in the fourth step, longer-term face-to-face psychotherapy and antidepressant medication might be considered.

- Patients are monitored by one person, a care manager, who is responsible for the decision to step up to the next treatment and for continuity of care.

- The different treatments within the stepped-care model are evidence-based. Data on cost-effectiveness of the full model are still scarce, but we recently demonstrated that the incidence of new cases of depression and anxiety could be halved by introducing stepped care.

- Effects of web-based guided self-help could be enhanced by incorporating them in a stepped-care model.

MJA 2010; 192: S36-S39

adults (18-65 years) with subthreshold and major depression and anxiety disorders. ${ }^{22,23}$ Both projects were conducted in primary care settings and had stepped-care interventions in which guided self-help was one of the main care elements.

\section{Stepped care: general layout}

In establishing a stepped-care project, it must first be determined which health care professionals need to contribute to the service. In both our research projects, we included psychiatric nurses who were used to working within a primary care clinic. Normally, they assist the general practitioner in diagnosing mental health problems and perform brief psychological interventions. ${ }^{24}$ In the context of our stepped-care projects, they were appointed as care managers and were responsible for monitoring the patients, making sure patients were invited for the next intervention, and for carrying out part of the interventions. They were trained for 2 days and we organised weekly supervision sessions. 
An important feature of stepped care is monitoring patients' improvements after each intervention. The duration of each of the interventions needs to be long enough to enable change, but it should not be too long. If the intervention does not lead to recovery, the patient needs to proceed to the next step as quickly as possible. We regard 6 to 8 weeks as the minimum time interval for any intervention (used in the project with younger adults) and 12 weeks as the maximum (used in the project with older adults). Monitoring could be based on clinical assessments, but both our research projects used standardised questionnaires because they are quick to complete and provide standardised measures of recovery. Several well validated depression questionnaires are available for this purpose. ${ }^{25}$ We used the Quick Inventory of Depressive Symptomatology (QIDS) in the project with younger adults and the Center for Epidemiologic Studies Depression Scale (CES-D) in the project with older adults. ${ }^{26-29}$

\section{Patients in the stepped-care model}

In the stepped-care trial of older adults, only patients with subthreshold disorders were included. We think that psychological disorders such as depressive disorders exist on a continuum rather than in separate categories. It is important to treat subthreshold depression because it causes a substantial loss of quality of life. ${ }^{30}$ Furthermore, treating subthreshold depression might prevent the disorder becoming full-blown. ${ }^{31}$ This way of thinking of mental disorders as being on a continuum, and about optimal treatment in different phases of the disease, can also be applied to other disorders. $^{32}$

In the trial of younger adults, all patients were included, from those with subthreshold disorders to those with severe depression. However, patients with severe depression were referred directly to the fourth step. Depression was considered to be severe when the patient had a major depressive episode and was unable to function adequately in daily life. Functioning was assessed with the Work and Social Adjustment Scale (WSAS), ${ }^{33}$ which is a simple fouritem scale measuring impairments related to work, home management, leisure activities, and relationships with others. Patients who performed poorly on at least three WSAS domains were referred to the fourth step. In our trials, this assessment of depression severity was performed by the researchers but, in regular care, it should be done by the patient's GP.

\section{First step: watchful waiting}

It is estimated that $50 \%$ of patients with a depressive episode recover spontaneously within 3 months. ${ }^{34}$ Therefore, in most clinical guidelines for depression (eg, Dutch and British guidelines ${ }^{35,36}$ ), "watchful waiting" is recommended as a first step in depression care. In concordance with these guidelines, we included watchful waiting as the first step in both our stepped-care projects. Duration was the same as for the other steps (6-8 weeks and 12 weeks in the trials with younger and older adults, respectively).

\section{Second step: guided self-help and internet-based interventions}

Self-help therapies are often based on cognitive behaviour therapy (CBT). In the Netherlands, the CBT-based course "Coping With Depression" has been converted into a self-help version. ${ }^{37}$ The self- help version is available in both book and web-based formats and consists of eight weekly lessons. ${ }^{38,39}$ It aims to improve social skills, to increase pleasant activities, to challenge depressogenic and anxiogenic thinking, and to improve coping with problems. This self-help course was offered in book format in our steppedcare trial with older people.

In the stepped-care trial with younger adults, we used a self-help course based on problem-solving therapy (PST) that consisted of five weekly lessons. In an earlier trial, we had demonstrated its effectiveness for people in the general population. ${ }^{40}$ The general idea of PST is that participants learn to regain control over their lives by determining what really matters to them and by learning a systematic approach to solving their problems. The advantage of PST is that it is a generic approach, which means that it is suitable for patients with different types of psychological problems and for patients with comorbidity. In our trial, participants could choose between a book or a web-based format of the course.

When delivering self-help interventions, it is recommended to offer some type of support to prevent drop-out. This support should be aimed at motivating patients and assisting them to master the self-help techniques. Support can be delivered face-toface, by telephone or online, and is provided by the care manager. It takes about 15 minutes a week to deliver web-based feedback for one participant.

\section{Third step: brief face-to-face therapy}

In both our trials, the care manager working with the GP provided brief face-to-face therapy, using PST, as the third step. PST, the therapy that was also used for one of our self-help interventions, is the most suitable form of face-to-face therapy because it is short (5-7 sessions of 30-45 minutes each), easy to understand (focuses on daily problems) and can be administered by non-mental health specialists such as nurses. It has been specifically developed for use in busy and time-constrained primary care settings. ${ }^{41}$ The literature shows that PST can be an effective treatment for depression, although the varying results warrant more research to ascertain the conditions and subjects in which these positive effects are realised. ${ }^{42,43}$

\section{Fourth step: longer-term face-to-face therapy and/or antidepressant medication}

Patients who do not recover after web-based self-help and brief face-to-face therapy need to step up to the next level of care: antidepressants, longer-term, evidence-based face-to-face therapy, or a combination of both. In this phase, it is important that a more detailed diagnostic interview is performed to determine why the former interventions did not work and what is needed. Patients might be referred to the GP (as was done in our stepped-care project with older adults) or directly to a mental health specialist (as was done in our stepped-care project with younger adults).

\section{Effects of our stepped-care trials}

Our stepped-care project involving younger adults is still in progress, with the results expected to be available in 2010. The stepped-care project for older adults with subthreshold depression demonstrated large preventive effects: the 12-month incidence of depression and anxiety was 24\% in the control group and $12 \%$ in the stepped-care group (relative risk, 0.49; 95\% CI, 0.24-0.98). 


\section{Discussion}

Stepped-care models such as the one we have described here might be a cost-effective way to improve depression management. Many GPs already implicitly work according to stepped-care principles, as they try to provide the least burdensome treatment that is most likely to induce significant health gains. However, there are a number of differences between this practice and the stepped-care model we propose. First, a mental health worker (such as a nurse) needs to be introduced into the primary care setting. A recent review demonstrated beneficial effects of on-site mental health workers, regardless of their background or the way care is organised. ${ }^{44}$ Second, minimal interventions such as guided self-help and internet-based interventions need to be introduced. The evidence on effectiveness of these for depression and anxiety disorders is so compelling that they are recommended in the United Kingdom by the National Institute for Health and Clinical Excellence for administering within the National Health Service. ${ }^{45}$ Particularly in the treatment of depression and anxiety, many excellent internet-based interventions have been developed, ${ }^{17,40,46}$ and there is no doubt that this type of intervention will become an important way of delivering minimal interventions to depressed and anxious patients.

New models of care delivery can only be implemented successfully when both providers and patients benefit from them. Results from Project IMPACT (Improving Mood, Promoting Access to Collaborative Treatment), a large trial on collaborative disease management for late-life depression in primary care, has shown that $93 \%$ of physicians using the new care-delivery model were very or somewhat satisfied, compared with $61 \%$ of physicians in the care-as-usual group. ${ }^{47}$ They identified proactive patient followup and patient education as the most helpful components of the new model. In the UK, stepped-care, evidence-based psychological treatments are implemented in routine care through the Improving Access to Psychological Therapies program. Observational studies on large numbers of patients show considerable effects on depression and anxiety of low-intensity, stepped-care treatments within a collaborative care system. ${ }^{48}$

One element of debate with respect to stepped care is which patients to include. There seems to be a general perception that minimal interventions should only be offered to patients with subthreshold or mild major depression. ${ }^{49,50}$ However, research involving patients with more severe symptoms is lacking. One solution is to supplement web-based guided self-help with medication for patients with severe depression. ${ }^{50}$ More research is needed to determine the effects of self-help in patients with more severe depression and to establish optimal cut-offs. Another point of consideration is whether to include patients with comorbid disorders. As about $60 \%$ of all patients with a major depressive disorder also suffer from an anxiety disorder, ${ }^{51}$ and because it is difficult to distinguish the two disorders without an extensive diagnostic interview, we think it is worthwhile to aim the steppedcare program and web-based self-help therapy at both disorders. Our web-based PST self-help course is very useful for this purpose because it is generic.

There are also some potential drawbacks in offering interventions according to stepped-care principles. If people do not respond adequately to the self-help intervention, they may experience a sense of failure that might reduce their motivation to start another treatment. Moreover, especially when the brief face-to-face treatment is based on the same principles as the self-help treatment (eg, PST), patients might experience each step as simply being "more of the same". If they did not respond to self-help, they may not respond to brief face-to-face therapy either. To minimise this risk, it is important to acknowledge that the stepped-care model should be seen as a treatment guideline. If patients indicate that they are not really motivated for a specific type of treatment, or they feel that it is not helping them, it might be wise to step up to the next treatment more quickly.

Current problems in depression management call for alternative methods of treatment delivery. Stepped care with web-based, guided self-help as an initial treatment step may be a useful and cost-efficient way of doing this. Our first results on stepped care for older patients with subthreshold depression are very promising, with more results on younger adults and costs to become available soon.

\section{Competing interests}

None identified.

\section{Author details}

Annemieke van Straten, PhD, Associate Professor ${ }^{1,2}$

Wike Seekles, MSc, PhD Student ${ }^{1,2}$

Nelleke J van 't Veer-Tazelaar, MA, PhD Student ${ }^{3,2}$

Aartjan TF Beekman, MD, PhD, Head of Department ${ }^{4,2}$

Pim Cuijpers, PhD, Head of Department ${ }^{1,2}$

1 Department of Clinical Psychology, VU University Amsterdam, Amsterdam, The Netherlands.

2 EMGO Institute for Health and Care Research, VU University Medical Center Amsterdam, Amsterdam, The Netherlands.

3 Department of General Practice, VU University Medical Center

Amsterdam, Amsterdam, The Netherlands.

4 Department of Psychiatry, VU University Medical Center Amsterdam, Amsterdam, The Netherlands.

Correspondence: a.van.straten@psy.vu.nl

\section{References}

1 Lopez AD, Mathers CD, Ezzati M, et al. Global and regional burden of disease and risk factors, 2001: systematic analysis of population health data. Lancet 2006; 367: 1747-1757.

2 Kruijshaar ME, Hoeymans N, Spijker J, et al. Has the burden of depression been overestimated? Bull World Health Organ 2005; 83: 443-448.

$3 \mathrm{Bijl}$ RV, de Graaf R, Hiripi E, et al. The prevalence of treated and untreated mental disorders in five countries. Health Aff (Millwood) 2003; 22: 122 133.

4 Wang PS, Aguilar-Gaxiola S, Alonso J, et al. Use of mental health services for anxiety, mood, and substance disorders in 17 countries in the WHO world mental health surveys. Lancet 2007; 370: 841-850.

5 Bebbington PE, Meltzer H, Brugha TS, et al. Unequal access and unmet need: neurotic disorders and the use of primary care services. Psychol Med 2000; 30: 1359-1367.

6 Andrews G, Issakidis C, Sanderson K, et al. Utilising survey data to inform public policy: comparison of the cost-effectiveness of treatment of ten mental disorders. Br J Psychiatry 2004; 184: 526-533.

7 Neumeyer-Gromen A, Lampert T, Stark K, Kallischnigg G. Disease management programs for depression: a systematic review and metaanalysis of randomized controlled trials. Med Care 2004; 42: 1211-1221.

8 Gilbody S, Bower P, Fletcher J, et al. Collaborative care for depression: a cumulative meta-analysis and review of longer-term outcomes. Arch Intern Med 2006; 166: 2314-2321.

9 Black DR, Threlfall WE. A stepped approach to weight control: a minimal intervention and a bibliotherapy problem-solving program. Behav Ther 1986; 17: 144-157.

10 Treasure J, Schmidt U, Troop N, et al. Sequential treatment for bulimia nervosa incorporating a self-care manual. Br J Psychiatry 1996; 168: 9498. 


\section{DELIVERING TIMELY INTERVENTIONS: THE IMPACT OF THE INTERNET ON MENTAL HEALTH}

11 Wilson GT, Vitousek KM, Loub KL. Stepped care treatment for eating disorders. J Consult Clin Psychol 2000; 68: 564-572.

12 Newman MG. Recommendations for a cost-offset model of psychotherapy allocation using generalized anxiety disorder as an example. $J$ Consult Clin Psychol 2000; 68: 549-555.

13 Otto MW, Pollack MH, Maki KM. Empirically supported treatments for panic disorder: costs, benefits, and stepped care. J Consult Clin Psychol 2000; 68: 556-563.

14 Sobell MB, Sobell LC. Stepped care as a heuristic approach to the treatment of alcohol problems. J Consult Clin Psychol 2000; 68: 573-579.

15 Bower P, Gilbody S. Stepped care in psychological therapies: access, effectiveness and efficiency. Narrative literature review. Br J Psychiatry 2005; 186: 11-17.

16 Spek V, Cuijpers P, Nyklícek I, et al. Internet-based cognitive behaviour therapy for symptoms of depression and anxiety: a meta-analysis. Psychol Med 2007; 37: 319-328.

17 Christensen H, Griffiths KM, Jorm AF. Delivering interventions for depression by using the internet: randomised controlled trial. BMJ 2004; 328: 265.

18 Cuijpers P. Bibliotherapy in unipolar depression: a meta-analysis. J Behav Ther Exp Psychiatry 1997; 28: 139-147.

19 McKendree-Smith NL, Floyd M, Scogin FR. Self-administered treatments for depression: a review. J Clin Psychol 2003; 59: 275-288.

20 Hickie IB, Davenport TA, Luscombe GM, et al. Practitioner-supported delivery of internet-based cognitive behaviour therapy: evaluation of the feasibility of conducting a cluster randomised trial. Med J Aust 2010; 192 (11 Suppl): S31-S35

21 Bower P, Richards D, Lovell K. The clinical and cost-effectiveness of selfhelp treatments for anxiety and depressive disorders in primary care: a systematic review. Br J Gen Pract 2001; 51: 838-845.

22 van 't Veer-Tazelaar PJ, van Marwijk HWJ, van Oppen P, et al. Steppedcare prevention of anxiety and depression in late life: a randomized controlled trial. Arch Gen Psychiatry 2009; 66: 297-304.

23 Seekles W, van Straten A, Beekman A, et al. Stepped care for depression and anxiety: from primary care to specialized mental health care: a randomised controlled trial testing the effectiveness of a stepped care program among primary care patients with mood or anxiety disorders. BMC Health Serv Res 2009; 9: 90.

24 Bower P, Gilbody S. Managing common mental health disorders in primary care: conceptual models and evidence base. BMJ 2005; 330: 839842.

25 Joiner TE Jr, Walker RL, Pettit JW, et al. Evidence-based assessment of depression in adults. Psychol Assess 2005; 17: 267-277.

26 Rush AJ, Trivedi MH, Ibrahim HM, et al. The 16-Item Quick Inventory of Depressive Symptomatology (QIDS), Clinician Rating (QIDS-C), and SelfReport (QIDS-SR): a psychometric evaluation in patients with chronic major depression. Biol Psychiatry 2003; 54: 573-583.

27 Trivedi MH, Rush AJ, Ibrahim HM, et al. The Inventory of Depressive Symptomatology, Clinician Rating (IDS-C) and Self-Report (IDS-SR), and the Quick Inventory of Depressive Symptomatology, Clinician Rating (QIDS-C) and Self-Report (QIDS-SR) in public sector patients with mood disorders: a psychometric evaluation. Psychol Med 2004; 34: 73-82.

28 Radloff LS. The CES-D scale: a self-report depression scale for research in the general population. Appl Psychol Meas 1977; 1: 385-401.

29 Beekman ATF, Deeg DJH, Van Limbeek J, et al. Criterion validity of the Center for Epidemiologic Studies Depression scale (CES-D): results from a community-based sample of older subjects in the Netherlands. Psychol Med 1997; 27: 231-235.

30 Cuijpers P, de Graaf R, van Dorsselaer S. Minor depression: risk profiles, functional disability, health care use and risk of developing major depression. J Affect Disord 2004; 79: 71-79.
31 Cuijpers $P$, van Straten A, Smit F, et al. Preventing the onset of depressive disorders: a meta-analytic review of psychological interventions. Am J Psychiatry 2008; 165: 1272-1280.

32 McGorry PD, Yung AR, Pantelis C, Hickie IB. A clinical trials agenda for testing interventions in earlier stages of psychotic disorders. Med J Aust 2009; 190 (4 Suppl): S33-S36.

33 Mundt JC, Marks IM, Shear MK, Greist JH. The Work and Social Adjustment Scale: a simple measure of impairment in functioning. $\mathrm{Br} J$ Psychiatry 2002; 180: 461-464.

34 Spijker J, de Graaf R, Bijl RV, et al. Duration of major depressive episodes in the general population: results from the Netherlands Mental Health Survey and Incidence Study (NEMESIS). Br J Psychiatry 2002; 181: 208213.

35 National Committee for the Development of Multidisciplinary Guideline. Multidisciplinary guideline for depression. Utrecht: Trimbos-instituut, 2005.

36 National Institute for Health and Clinical Excellence. Clinical guideline 23. Depression: management of depression in primary and secondary care. London: NICE, 2004.

37 Lewinsohn PM, Antonuccio DO, Steinmetz JL, Teri L. The Coping With Depression Course: a psychoeducational intervention for unipolar depression. Eugene, Ore: Castalia Publishing Co, 1984.

38 Spek V, Nyklícek I, Smits N, et al. Internet-based cognitive behavioural therapy for subthreshold depression in people over 50 years old: a randomized controlled clinical trial. Psychol Med 2007; 37: 1797-1806.

39 Haringsma R, Engels GI, Cuijpers P, Spinhoven P. Effectiveness of the Coping With Depression (CWD) course for older adults provided by the community based mental health care system in the Netherlands: a randomized controlled field trial. Int Psychogeriatr 2006; 18: 307-325.

40 van Straten A, Cuijpers P, Smits N. Effectiveness of a web-based self-help intervention for symptoms of depression, anxiety and stress: randomized controlled trial. J Med Internet Res 2008; 10 (1): e7.

41 Mynors-Wallis L. Problem-solving treatment for anxiety and depression: a practical guide. Oxford: Oxford University Press, 2005.

42 Cuijpers P, van Straten A, Warmerdam L. Problem solving therapies for depression: a meta-analysis. Eur Psychiatry 2007; 22: 9-15.

43 Huibers MJ, Beurskens A, Bleijenberg G, van Schayck CP. Psychosocial interventions by general practitioners. Cochrane Database Syst Rev 2007; (3): CD003494.

44 Harkness EF, Bower PJ. On-site mental health workers delivering psychological therapy and psychosocial interventions to patients in primary care: effects on the professional practice of primary care providers. Cochrane Database Syst Rev 2009; (1): CD000532.

45 Marks IM, Cavanagh K, Gega L. Computer-aided psychotherapy: revolution or bubble? Br J Psychiatry 2007; 191: 471-473.

46 Andersson G, Bergström J, Hollandare F, et al. Internet-based self-help for depression: randomised controlled trial. Br J Psychiatry 2005; 187: 456-461.

47 Levine S, Unutzer J, Yip JY, et al. Physicians' satisfaction with a collaborative disease management program for late-life depression in primary care. Gen Hosp Psychiatry 2005; 27: 383-391.

48 Richards DA, Suckling R. Improving access to psychological therapies: Phase IV prospective cohort study. Br J Clin Psychol 2009; 48: 377-396. DOI: $10.1348 / 014466509 \times 405178$.

49 Lovell K, Richards D. Multiple access points and levels of entry (MAPLE): ensuring choice, accessibility and equity for CBT services. Behav Cogn Psychother 2000; 28: 379-391.

50 Scogin FR, Hanson A, Welsh D. Self-administered treatment in steppedcare models of depression treatment. J Clin Psychol 2003; 59: 341-349.

51 Kessler RC, Berglund P, Demler O, et al. The epidemiology of major depressive disorder: results from the National Comorbidity Survey Replication (NCS-R). JAMA 2003; 289: 3095-3105.

(Received 3 Sep 2009, accepted 19 Jan 2010) 